\title{
Induction of Reparative Dentin Formation on Exposed Dental Pulp by Dentin Phosphophoryn/Collagen Composite
}

\author{
Toshiyuki Koike, Mohammad Ali Akbor Polan, Masanobu Izumikawa, and Takashi Saito
}

Division of Clinical Cariology and Endodontology, Department of Oral Rehabilitation, School of Dentistry, Health Sciences University of Hokkaido, 1757 Tobetsu, Hokkaido 061-0293, Japan

Correspondence should be addressed to Takashi Saito; t-saito@hoku-iryo-u.ac.jp

Received 3 February 2014; Accepted 25 February 2014; Published 6 April 2014

Academic Editor: Yoshihiko Hayashi

Copyright (C) 2014 Toshiyuki Koike et al. This is an open access article distributed under the Creative Commons Attribution License, which permits unrestricted use, distribution, and reproduction in any medium, provided the original work is properly cited.

The ultimate goal of vital pulp therapy is to regenerate rapidly dentin possessing an excellent quality using a biocompatible, bioactive agent. Dentin phosphophoryn (DPP), the most abundant noncollagenous polyanionic protein in dentin, cross-linked to atelocollagen fibrils was applied to direct pulp capping in rats. After 1, 2, and 3 weeks, the teeth applied were examined on the induction of reparative dentin formation and the response of pulp tissue, compared to calcium hydroxide-based agent conventionally used. The reparative dentin formation induced by DPP/collagen composite was more rapid than by calcium hydroxide. In the morphometrical analysis, the formation rate of reparative dentin by DPP/collagen composite was approximately the same as that by calcium hydroxide at 3 weeks. Nevertheless, the compactness of reparative dentin formed by DPP/collagen composite was much superior to what resulted from calcium hydroxide. Also, DPP/collagen composite showed high covering ability of exposed pulp. Moreover, DPP/collagen composite led only to slight pulp inflammation at the beginning whereas calcium hydroxide formed necrotic layer adjacent to the material and induced severe inflammation in pulp tissue at 1 week. The present study demonstrates a potential for DPP/collagen composite as a rapid biocompatible inducer for the formation of reparative dentin of excellent quality in rats.

\section{Introduction}

Direct pulp capping using calcium hydroxide-based agent has been a standard therapy for years. However, long-term studies have shown results to be variable and unpredictable [1-3]. Thus, clinical success rate of the therapy has not satisfied dentists. The agent does not provide close adaptation to dentin, does not promote consistent odontoblast differentiation, and has been shown to be cytotoxic in cell cultures, leading to the fact that the resultant reparative dentin formation can be characterized by tunnel defects [4-6]. Tunnel defects within reparative dentin may provide a pathway for the penetration of microorganisms to develop secondary infection of pulp tissue. Furthermore, low mechanical strength and pulpal resorption are potential disadvantage of the agent $[5,7]$. Therefore, a biocompatible, bioactive agent that rapidly induces reparative dentin formation possessing a good quality is required to improve the clinical success rate of vital pulp therapy.
Dentin phosphophoryn (DPP), a member of small integrin-binding ligand N-linked glycoproteins (SIBLING) family, is the most abundant of the noncollagenous polyanionic proteins in dentin. DPP is dentin sialophosphoprotein (DSPP) gene product $[8,9]$. It is predominantly expressed in odontoblasts and is known to be a marker of the differentiation of pulp cells into odontoblasts [10-12]. It contains the RGD motif at position of 26 from N-terminal and the repeating sequence of (asparatic acid-phosphoserine-phosphoserine) ${ }_{\mathrm{n}}$ as its characteristic domains [13]. Aspartic acid and serine account for at least $75 \%$ of all amino acid sequences, and $85 \%-90 \%$ of the serine residues are phosphorylated. DPP is supported by in vitro mineralization data showing that DPP is an important initiator and modulator for the formation and growth of hydroxyapatite crystals due to many negatively charged regions [14-19]. In particular, DPP covalently cross-linked on collagen fibrils has high potential to nucleate hydroxyapatite according to the measure of interfacial energy [17-19]. 
Recently, DPP has been reported to induce the differentiation of human mesenchymal stem cells into osteoblasts as its RGD motif binds with $\alpha \mathrm{v} \beta 3$ integrin on the cell surface followed by the activation of the MAP kinase and SMAD signaling pathway $[20,21]$. Moreover, we demonstrated that DPP promotes not only differentiation or calcification but also cell migration by acting on integrin on the surface of pulp cells via its RGD motif in vitro [22]. However, the potential of DPP for dentin regeneration in animal model has not still been clear although there is much evidence on DPP in vitro ability.

The aim of this study was to investigate effects of DPP cross-linked to type I atelocollagen fibrils as a scaffold placed on experimentally created pulp exposures in first molar in rats on induction of reparative dentin formation and response of pulp tissue, compared to calcium hydroxide-based agent conventionally used.

\section{Materials and Methods}

2.1. Preparation of DPP Cross-Linked to Type I Atelocollagen Fibrils. The DPP was prepared from molar teeth extracted from 8-9-month-old porcine jaws following the method described by Butler [23]. The preparation was carried out at $4^{\circ} \mathrm{C}$ in solutions to which protease inhibitors $(100 \mathrm{mM}$ 6-aminohexanoic acid, $5 \mathrm{mM}$ benzamidine- $\mathrm{HCl}$, and $1 \mathrm{mM}$ phenylmethylsulfonyl fluoride) were added. The DPP was extracted with calcium precipitation method and purified through the anion exchange chromatography step.

Type I atelocollagen fibrils, from which telopeptides known to be antigen had been removed, were used as a carrier of DPP in this study. The DPP was cross-linked to porcinederived type I atelocollagen fibrils (collagen sponge, Nitta gelatin, Osaka, Japan) with divinylsulfone (Sigma Chemical, St. Louis, MO, USA) [24]. To remove unreacted divinylsulfone and DPP that was not covalently bound, the substrates were washed with $0.5 \mathrm{M} \mathrm{NaCl}, 0.05 \mathrm{M}$ Tris- $\mathrm{HCl}$, and $\mathrm{pH}$ 7.4, ten times and then washed with distilled water. The composite was analyzed for phosphate analysis [25] after alkaline hydrolysis to determine the amount of DPP crosslinked to type I atelocollagen fibrils.

2.2. Surgical Procedure. All animal procedures were in accordance with the guidelines of the Animal Care Committee of the Health Sciences University of Hokkaido. Eight-week-old male Wistar rats (Hokudo, Sapporo, Japan) were used in this study. Animals were anaesthetized with an intraperitoneal injection of pentobarbital $(40 \mathrm{mg} / \mathrm{kg})$. The vital pulp tissue was exposed by drilling the mesial buccal cusp of the maxilla first molar using a sterile, round steel bur (number 014, Dentsply, York, PA, USA). The exposed pulp tissue was treated with $10 \%$ sodium hypochlorite and $3 \%$ hydrogen peroxide. Then, bleeding was controlled with sterile cotton pellets. Subsequently, the exposed pulp tissue was covered with $0.5 \mu \mathrm{g}$ of DPP covalently cross-linked to $29.5 \mu \mathrm{g}$ of type I atelocollagen fibrils (DPP-Col), type I atelocollagen alone (Col), and multi-Cal (Ca) (Pulpdent, Watertown, MA, USA), one of calcium hydroxide-based agents which have been conventionally used for direct pulp capping. Twentyone teeth were used in each group (DPP-Col, Col, and Ca) and were further divided into three groups on the basis of experimental period (1,2, and 3 weeks). Following the pulp capping, each cavity was immediately sealed with glassionomer cement (Hy-bond Glasionomer CX, Shofu, Koto, Japan) and was filled with single step bonding system (Clearfil S-3 Bond, Kuraray, Tokyo, Japan) and composite resin (Unifil flow, GC, Tokyo, Japan).

2.3. Histological Examination. At 1, 2, and 3 weeks after surgery, the animals were killed by overinhalation of diethyl ether. Following death, all experimental teeth and the adjacent alveolar bone were removed and fixed with $10 \%$ neutral buffered formalin for 24 hours. Then, specimens were

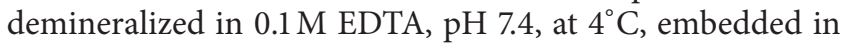
paraffin, sectioned at $4 \mu \mathrm{m}$, and subsequently stained with hematoxylin and eosin. The sections were then analyzed in a light microscope (Eclipse E400, Nikon, Tokyo, Japan).

\subsection{Morphometrical Analysis of Reparative Dentin Formed} and Evaluation of Pulp Tissue. First, the formation rate of reparative dentin, that is, relative area of reparative dentin formed adjacent to the cavity to the area of pulp chamber of crown in each experimental tooth, was assessed in the longitudinal section covering the central part of the experimental wound. The areas in the sections were measured by means of Image J (Wayne Rasband, MD, USA) in three sections in separate seven teeth. Next, the compactness of reparative dentin formed in the sections was measured based on the area of defect in reparative dentin and the total area of reparative dentin. The formation rate and compactness of reparative dentin were calculated for formula as follows [26]:

Formation rate $(\%)=$ total area of tertiary dentin/ coronal pulp chamber area $\times 100$

Compactness $(\%)=1-($ defects area + cells area $) /$ total area of tertiary dentin $\times 100$.

Then, the covering degrees of exposed pulp tissue with reparative dentin formed were assessed in four scores; 4 : completely covered, 3: almost covered (50\% and over covering at the pulp exposure surface), 2: partially covered (49\% and less covering at the pulp exposure surface), and 1: reparative dentin formation not observed. Moreover, the degrees of pulp inflammation induced by the materials were evaluated in four scores; 4: minimal inflammation (none or few scattered inflammatory cells present in the pulp at the exposure site, or same as normal dental pulp), 3: mild inflammation (some vasodilation of blood vessels, indicating mild hyperemia on the surface of the exposure site), 2: moderate inflammation (presence of weak vasodilation of blood vessels without infiltration of blood cells into the dental pulp, some inflammatory cells, such as polymorphonuclear leukocytes and neutrophils, observed), 1: severe inflammation (presence of strong vasodilation of blood vessels appearing as an abscess and significant inflammatory infiltration by polymorphonuclear leukocytes and neutrophils seen throughout the crown) [26]. 

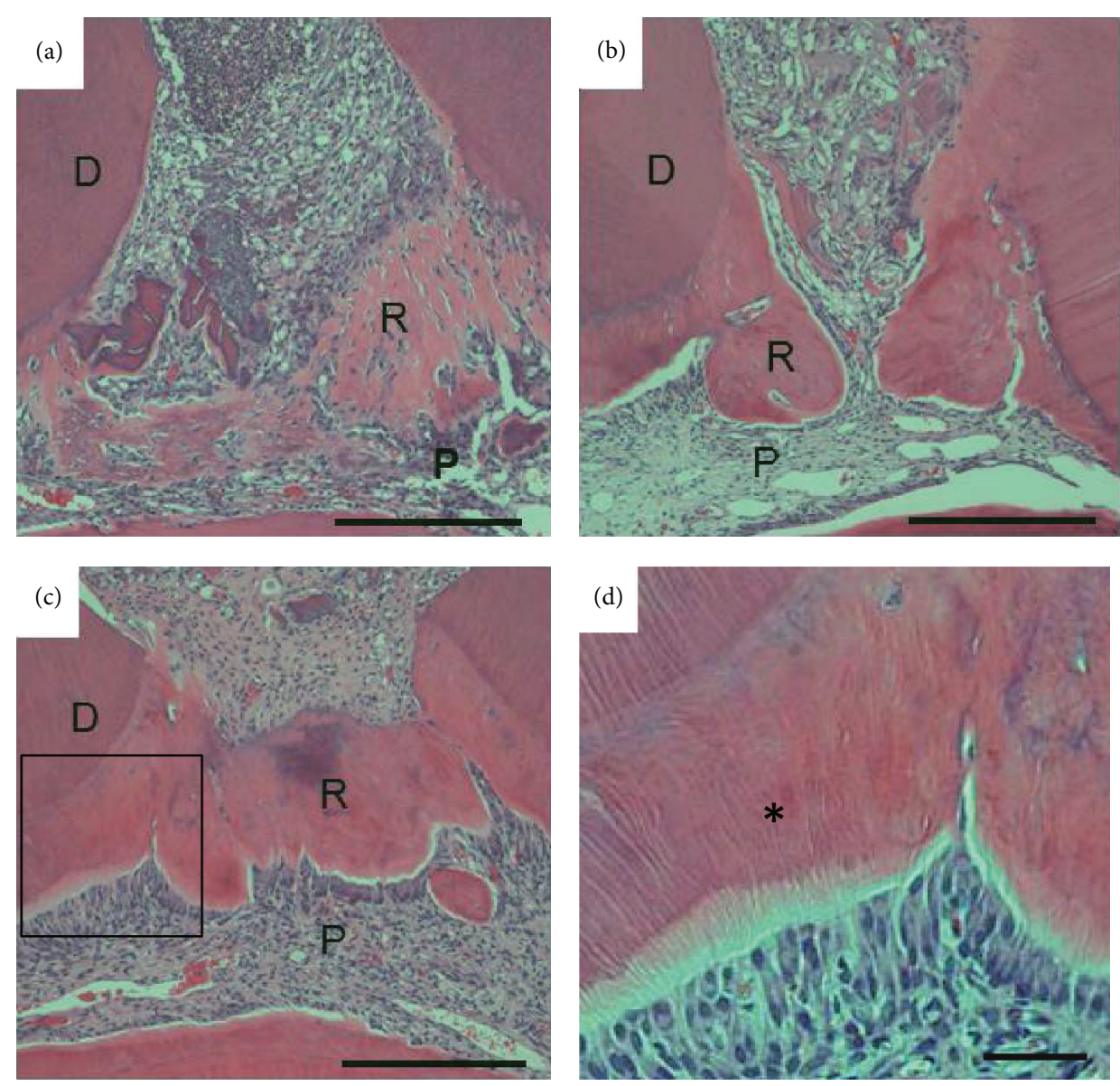

FIGURE 1: Micrographs showing upper first molars 1(a), 2(b), and 3 weeks ((c) and (d)) after treatment with DPP-Col. The compact reparative dentin was formed adjacent to the primary dentin at 2 weeks (b). The exposed pulp tissue was covered completely with reparative dentin at 3 weeks (c). Reparative dentin has dentinal tubules (asterisk) and regular arrangement of odontoblasts at 3 weeks ((d) higher magnification of the highlighted region in (c)). D: dentin, P: pulp tissue, and R: reparative dentin. Scale bar ((a), (b), and (c)) $=500 \mu \mathrm{m}$. Scale bar (d) $=100 \mu \mathrm{m}$.

2.5. Statistical Analysis. The results for the formation rate, the compactness of reparative dentin formed, and the degrees of pulp inflammation induced by the materials are expressed as means $\pm \mathrm{SD}$. They were analyzed by one-way analysis of variance with Tukey's multiple comparison test. Differences at $P<0.05$ were considered statistically significant.

\section{Results}

The formation rate of reparative dentin by every group increased with time (Figures 1-3). In DPP-Col group, the formation rate of reparative dentin was significantly higher, compared with that by Col group throughout the experimental period (Figure 4(a)). It was also significantly higher than that by Ca group at 1 and 2 weeks. However, no significant difference was noted at 3 weeks between groups DPP-Col and $\mathrm{Ca}$. In addition, the compactness of reparative dentin formed by DPP-Col group was significantly higher, compared with that formed by Ca group at 2 and 3 weeks (Figure 4(b)). In particular, it showed approximately $97 \%$ at 3 weeks. In DPP-Col group, reparative dentin possessing dentinal tubules was formed adjacent to the primary dentin at 3 weeks (Figures 1(c) and 1(d), asterisk). The exposed pulp tissue was covered completely with reparative dentin (Figures 1(c) and 4(c)) having regular arrangement of odontoblasts at 3 weeks (Figure 1(d)). The slight pulp inflammation was observed at 1 and 2 weeks, but no pulp inflammation was observed at 3 weeks (Figures 1 and 4(d)).

In Col group, reparative dentin formation was observed at 2 weeks, but reparative dentin did not cover exposed pulp tissue at even 3 weeks (Figures 2 and 4(c)). Moreover, the mild pulp inflammation was observed only at the early stage after operation (Figure $4(\mathrm{~d})$ ).

In $\mathrm{Ca}$ group, reparative dentin having considerable amount of gaps and tunnel defects was observed at 2 weeks (Figure 3(b), arrow). At 3 weeks, the reparative dentin covered exposed pulp tissue but still contained gaps and tunnel defects (Figure 4(c)). The arrangement of odontoblasts underneath reparative dentin formed by $\mathrm{Ca}$ group was not clear. The formation of necrotic layer adjacent to the material and severe inflammation in pulp tissue were observed at 1 week (Figure 3(a)). The mild pulp inflammation still remained at 3 weeks (Figures 3(c) and 4(d)). 

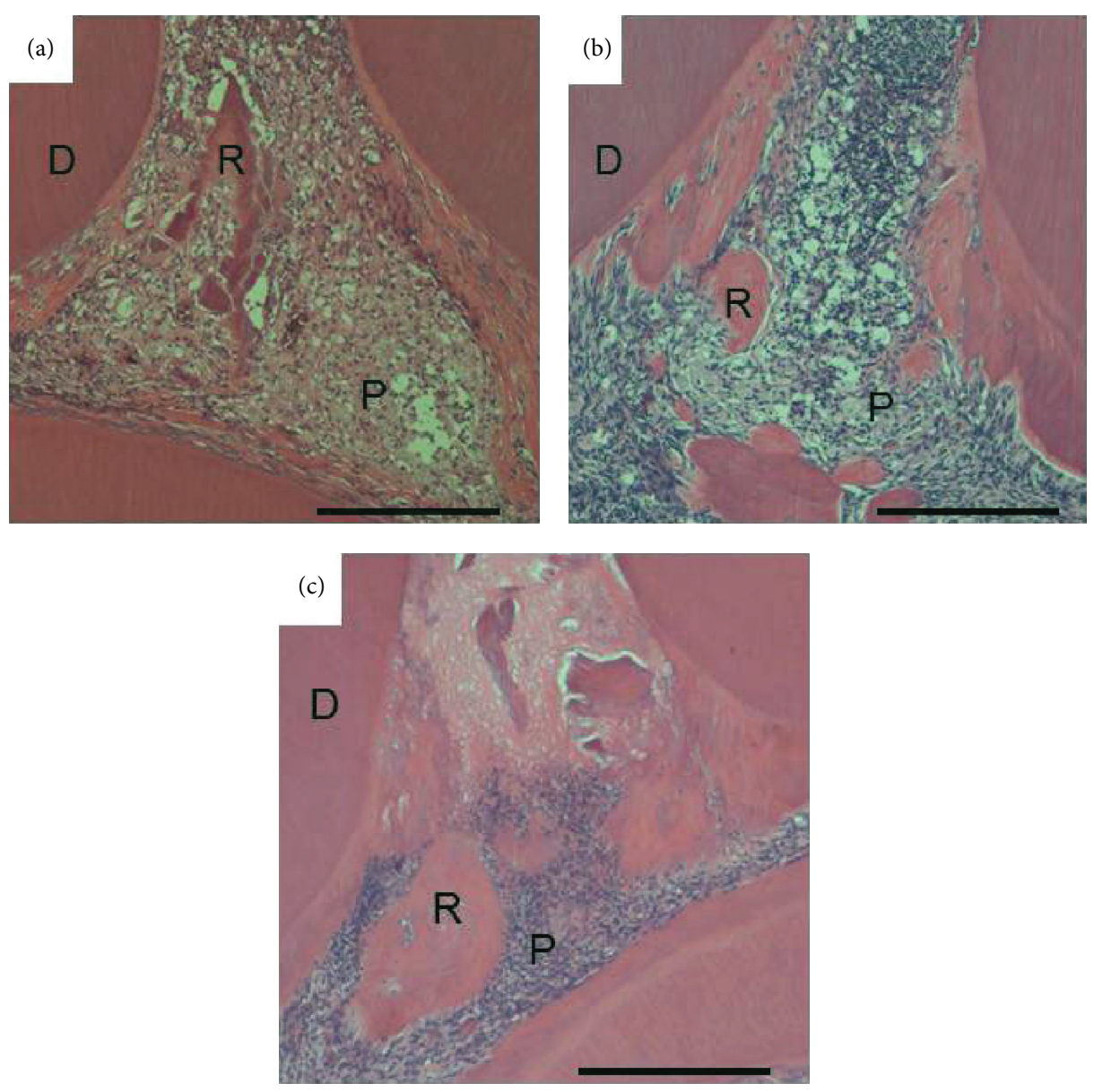

Figure 2: Micrographs showing upper first molars 1(a), 2(b), and 3 weeks (c) after treatment with Col. Reparative dentin is observed at 2 weeks but does not cover exposed pulp tissue even at 3 weeks. D: dentin, P: pulp tissue, and R: reparative dentin. Scale bar $=500 \mu \mathrm{m}$.

\section{Discussion}

It has been reported in clinical studies that the success rate of direct pulp capping with calcium hydroxide that has been used conventionally is approximately $60 \%$ even though it has been considered the standard therapy [27]. The clinical failures of direct pulp capping have led to the search for new therapeutic agents. The potential of bioactive agents such as dentin extracellular matrix molecules, for example, BMP-2, BMP-4, and BMP-7 (osteogenic protein-1: OP-1), dentin matrix protein-1 (DMP-1), matrix extracellular phosphoglycoprotein (MEPE), bone sialoprotein (BSP), and so forth, enamel matrix derivative, or stem cells is currently under study [11, 28-34]. Recently, mineral trioxide aggregate (MTA) was found to be an excellent pulp-capping agent because of its high biocompatibility [35]. However, dentin bridge formation by MTA was shown to be slower compared to calcium hydroxide [36]. In the clinical case, exposed pulp should be closed by the complete dentin bridge in a short period. The dentin extracellular matrix molecules are promising materials because of being involved in dentinogenesis during the development of teeth [37]. Therefore, they can bring out the potential of stem cells or progenitor cells located within the pulp, to the maximum, to proliferate, differentiate into odontoblast-like cells, and consequently produce the extracellular matrix, which will ultimately undergo mineralization.

In the present study, we confirmed DPP's high ability for dentin regeneration in animal model. After direct pulp capping with DPP cross-linked to type I collagen, biocompatible scaffold, the formation of reparative dentin was more rapid at the beginning than with calcium hydroxide. The rapid induction of reparative dentin reduces the chance of secondary infection of the pulp. No significant difference was found in the formation rate of reparative dentin between DPP crosslinked to collagen and calcium hydroxide at 3 weeks after operation. However, the compactness of reparative dentin formed by DPP cross-linked to collagen was significantly higher compared with that formed by calcium hydroxide at 2 and 3 weeks. In particular, the porous reparative dentin having tunnel defect was formed by calcium hydroxide. It has been reported that $89 \%$ of dentin bridges formed by calcium hydroxide contained tunnel defects [5]. The tunnel defects fail to provide a hermetic seal to the underlying pulp against recurring infection due to microleakage. This reparative dentin has a bone-like structure that does not possess odontoblast layer underneath it and consequently does 

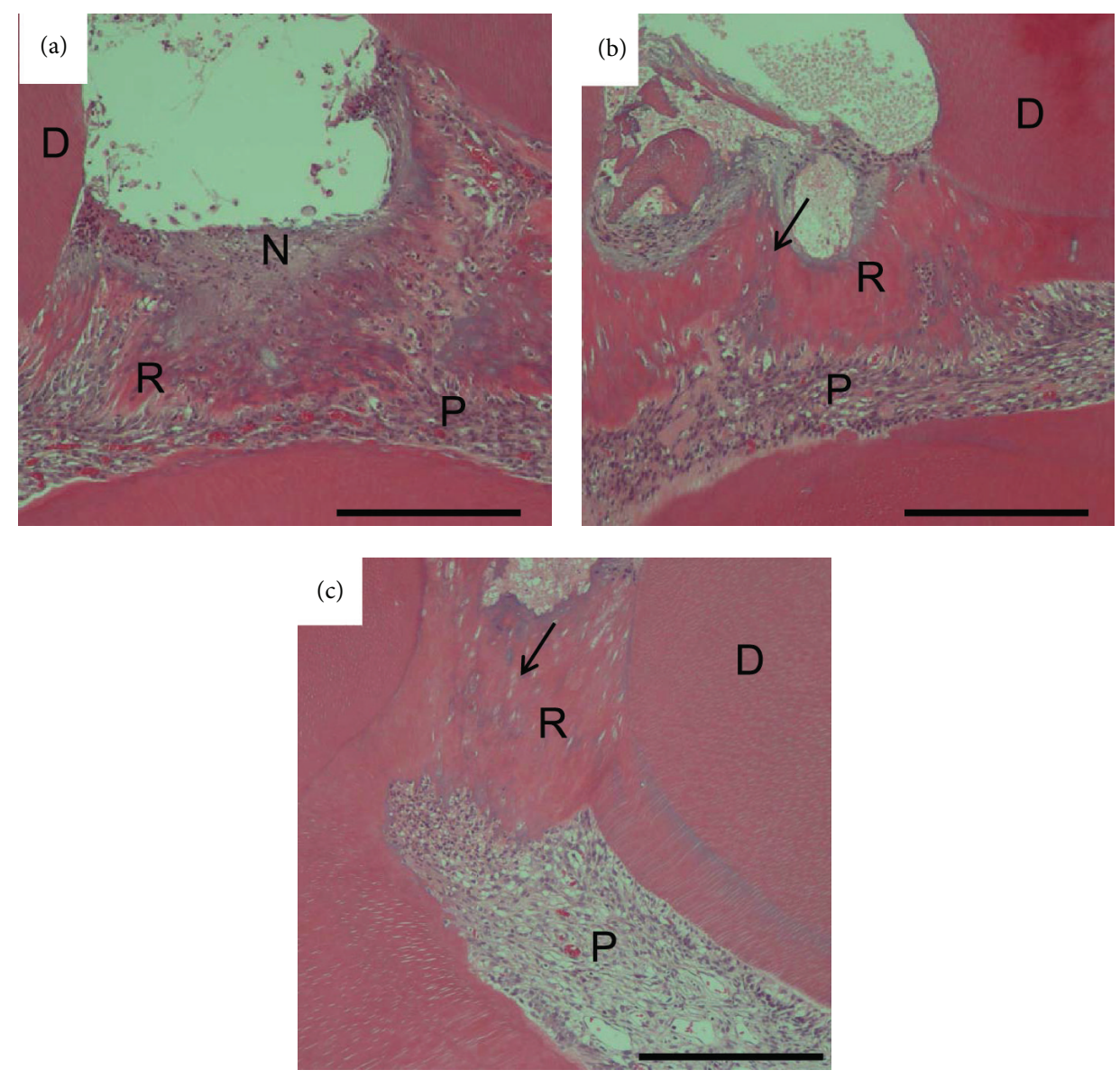

FIGURE 3: Micrographs showing upper first molars 1(a), 2(b), and 3 weeks (c) after treatment with Ca. Reparative dentin having gaps and tunnel defects was observed at 2 weeks (arrow). This dentin still contained gaps and tunnel defects at 3 weeks. A necrotic superficial layer (N) formed beneath the material. D: dentin, P: pulp tissue, and R: reparative dentin. Scale bar $=500 \mu \mathrm{m}$.

not contain dentinal tubules. In contrast, DPP immobilized to type I collagen induced the compact reparative dentin having regular arrangement of odontoblasts and dentinal tubules. This accounts for the quality difference of reparative dentin formed between DPP immobilized to type I collagen and calcium hydroxide. Thus, it was suggested that DPP induced differentiation from undifferentiated pulp cells into odontoblasts on type I collagen fibrils, suitable scaffolds for the cell differentiation, after the activation of the MAP kinase and SMAD signaling pathway [20, 21].

DPP immobilized to type I collagen caused only slight pulp inflammation at the early stage after operation due to the biocompatibility of collagen. The pulp exposure and implantation onto the pulp of biomaterials induce inflammation. Many studies report that the healing sequence includes an initial inflammation process [38-40]. In inflammatory phenomena, pulp cells express class II antigens [41]. They are implicated in the immune reaction. Immune cells such as dendritic cells and macrophages are crucial in the control of cell proliferation and apoptosis [42]. They contribute in the exposed pulp to resolve inflammatory process [43]. Then, the reparative process follows after inflammation. Therefore, inflammatory reaction may be another prerequisite for the repair process of dentin/pulp complex. On the other hand, calcium hydroxide formed necrosis layer underneath the agent due to its high alkalinity and also caused pulp inflammation. It has been reported that $41 \%$ of them were associated with recurring pulp inflammation or necrosis [5]. Thus, calcium hydroxide cannot be considered as a biocompatible agent although it is often used as a convenient low-cost material for vital pulp therapy.

In the present study, divinylsulfone was used for covalent cross-linking of DPP to type I collagen fibrils. It has been reported as a cross-linker of biomaterials in vitro and in vivo $[44,45]$. It is contained in the osteoarthritis knee pain relief treatment agent for clinical use [46]. Thus, DPP/collagen composite can be considered as a safe material. For clinical application of DPP/collagen, however, unreacted divinylsulfone should be removed from the composite completely after cross-linking procedure, due to its toxicity [47].

This is the first time that DPP/collagen composite showing stimulation of reparative dentin formation was reported. Our observation might help develop a reliable and safe vital pulp therapy. However, it is necessary to develop artificial materials such as recombinant protein and synthetic peptide of DPP for clinical trial for avoiding possible immune 


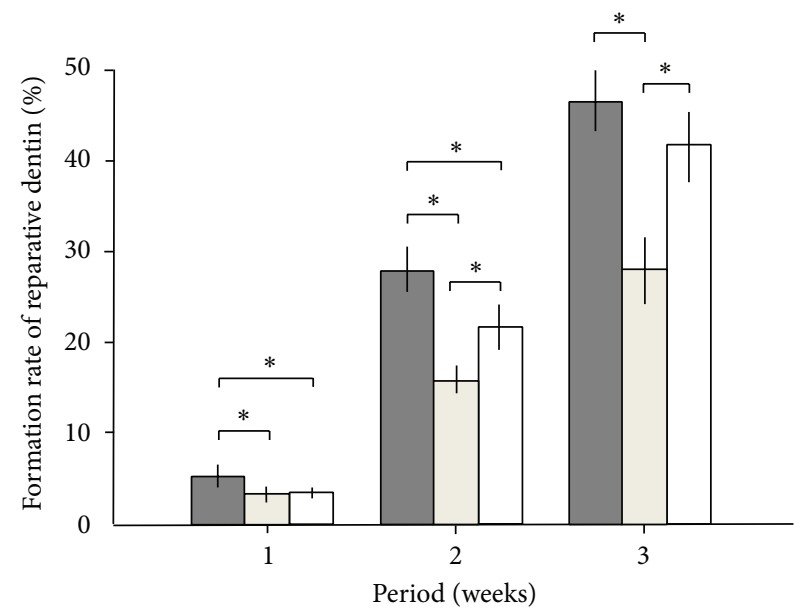

(a)

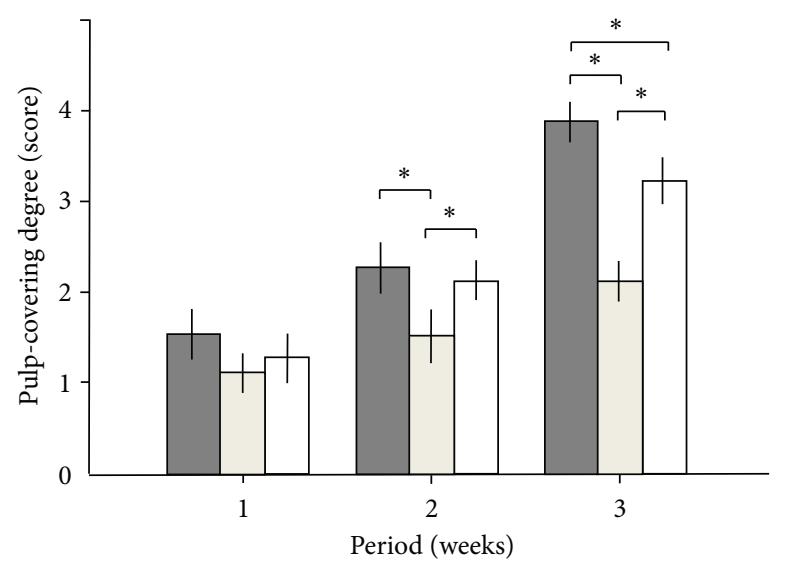

(c)

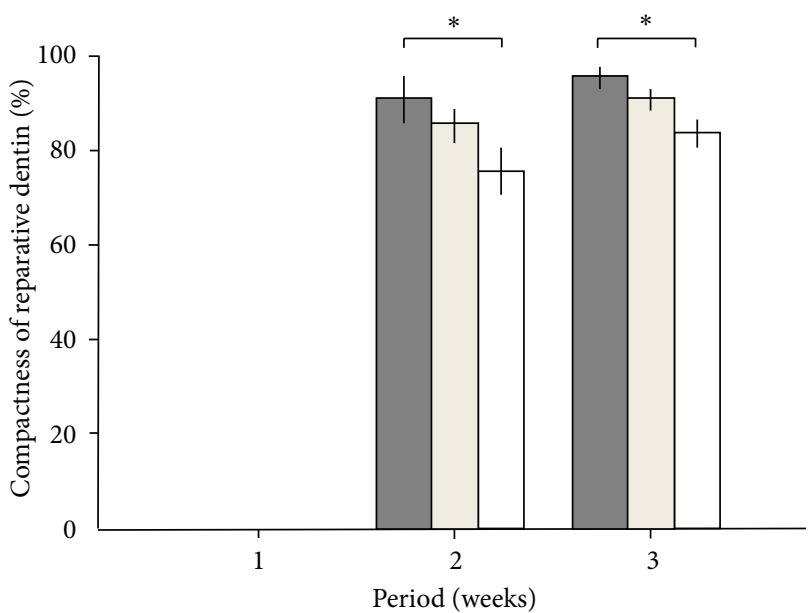

(b)

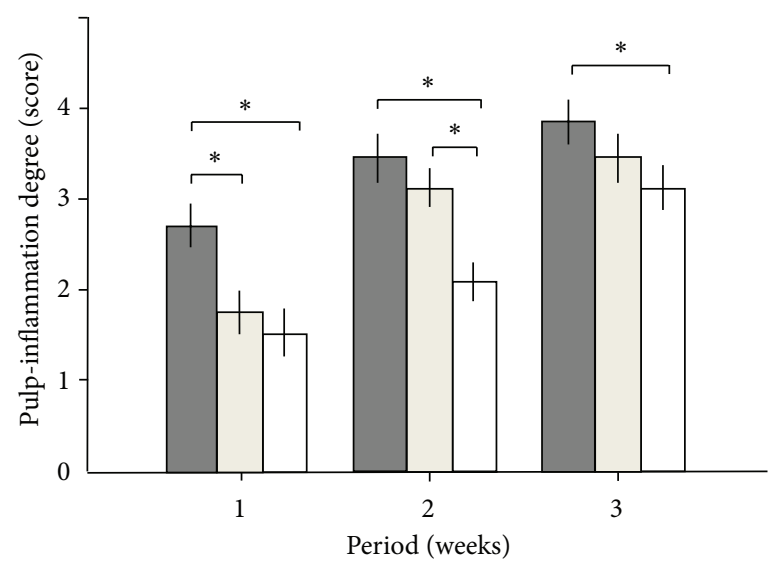

(d)

FIGURE 4: Evaluation of the reparative dentin and dental pulp tissue. (a) Formation rate of the reparative dentin (\%), (b) compactness of the reparative dentin (\%), (c) degree of coverage by the reparative dentin (score), and (d) inflammation degree of the dental pulp tissue (score). Gray columns: DPP-Col, beige columns: Col, and white columns: Ca. Twenty-one teeth were used in each group (DPP-Col, Col, and Ca) and were further divided into three groups on the basis of experimental period $\left(1,2\right.$, and 3 weeks). ${ }^{*} P<0.05$.

reaction and contamination of unidentified factors. Also, more investigations in long-term experiments will reveal more characteristics of DPP/collagen composite for dentin regeneration, and the further refinement of the collagen scaffold is necessary in order to utilize maximally the potential of DPP to stimulate the formation of reparative dentin as a hard tissue barrier. Moreover, it is crucial to understand the mechanisms underlining the process of pulp repair as well as dentin regeneration.

\section{Conclusion}

The present study demonstrates a potential for DPP/collagen composite as a rapid biocompatible inducer for the formation of reparative dentin of excellent quality in rats. Therefore, it is a promising biocompatible pulp capping material for the future.

\section{Disclosure}

The authors declare that this paper is original, has not been published before, and is not currently being considered for publication elsewhere. They confirmed that the paper has been approved by all named authors. They further confirm that the order of authors listed in the paper has been approved by all of them.

\section{Conflict of Interests}

The authors declare that there is no conflict of interests regarding the publication of this paper.

\section{Acknowledgment}

This work was supported by a Grant-in-Aid for Scientific Research from the Japanese Society for the Promotion of Science (Grant nos. 20390484 and 23390436). 


\section{References}

[1] P. Hørsted, B. Søndergaard, A. Thylstrup, K. El Attar, and O. Fejerskov, "A retrospective study of direct pulp capping with calcium hydroxide compounds," Endodontics \& Dental Traumatology, vol. 1, no. 1, pp. 29-34, 1985.

[2] L. J. Baume and J. Holz, "Long term clinical assessment of direct pulp capping," International Dental Journal, vol. 31, no. 4, pp. 251-260, 1981.

[3] C. R. Barthel, B. Rosenkranz, A. Leuenberg, and J. Roulet, "Pulp capping of carious exposures: treatment outcome after 5 and 10 years: a retrospective study," Journal of Endodontics, vol. 26, no. 9, pp. 525-528, 2000.

[4] U. Schröder, "Effects of calcium hydroxide-containing pulpcapping agents on pulp cell migration, proliferation, and differentiation," Journal of Dental Research, vol. 64, pp. 541-548, 1985.

[5] C. F. Cox, R. K. Sübay, E. Ostro, S. Suzuki, and S. H. Suzuki, "Tunnel defects in dentin bridges: their formation following direct pulp capping," Operative Dentistry, vol. 21, no. 1, pp. 411, 1996.

[6] W. E. Andelin, S. Shabahang, K. Wright, and M. Torabinejad, "Identification of hard tissue after experimental pulp capping using dentin sialoprotein (DSP) as a marker," Journal of Endodontics, vol. 29, no. 10, pp. 646-650, 2003.

[7] M. Hwas and J. L. Sandrik, "Acid and water solubility and strength of calcium hydroxide bases," The Journal of the American Dental Association, vol. 108, no. 1, pp. 46-48, 1984.

[8] H. H. Ritchie and L. Wang, "The presence of multiple rat DSPPP transcripts," Biochimica et Biophysica Acta-Gene Structure and Expression, vol. 1493, no. 1-2, pp. 27-32, 2000.

[9] K. Gu, S. Chang, H. H. Ritchie, B. H. Clarkson, and R. B. Rutherford, "Molecular cloning of a human dentin sialophosphoprotein gene," European Journal of Oral Sciences, vol. 108, no. 1, pp. 35-42, 2000.

[10] X. Wei, J. Ling, L. Wu, L. Liu, and Y. Xiao, "Expression of mineralization markers in dental pulp cells," Journal of Endodontics, vol. 33, no. 6, pp. 703-708, 2007.

[11] T. Saito, M. Ogawa, Y. Hata, and K. Bessho, "Acceleration effect of human recombinant bone morphogenetic protein-2 on differentiation of human pulp cells into odontoblasts," Journal of Endodontics, vol. 30, no. 4, pp. 205-208, 2004.

[12] W. E. Andelin, S. Shabahang, K. Wright, and M. Torabinejad, "Identification of hard tissue after experimental pulp capping using dentin sialoprotein (DSP) as a marker," Journal of Endodontics, vol. 29, no. 10, pp. 646-650, 2003.

[13] A. George, L. Bannon, B. Sabsay et al., "The carboxylterminal domain of phosphophoryn contains unique extended triplet amino acid repeat sequences forming ordered carboxylphosphate interaction ridges that may be essential in the biomineralization process," Journal of Biological Chemistry, vol. 271, no. 51, pp. 32869-32873, 1996.

[14] A. L. Boskey, M. Maresca, S. Doty, B. Sabsay, and A. Veis, "Concentration-dependent effects of dentin phosphophoryn in the regulation of in vitro hydroxyapatite formation and growth," Bone and Mineral, vol. 11, no. 1, pp. 55-65, 1990.

[15] A. George and J. Hao, "Role of phosphophoryn in dentin mineralization," Cells Tissues Organs, vol. 181, no. 3-4, pp. 232240, 2006.

[16] G. He, A. Ramachandran, T. Dahl et al., "Phosphorylation of phosphophoryn is crucial for its function as a mediator of biomineralization," Journal of Biological Chemistry, vol. 280, no. 39, pp. 33109-33114, 2005.
[17] T. Saito, A. L. Arsenault, M. Yamauchi, Y. Kuboki, and M. A. Crenshaw, "Mineral induction by immobilized phosphoproteins," Bone, vol. 21, no. 4, pp. 305-311, 1997.

[18] T. Saito, M. Yamauchi, and M. A. Crenshaw, "Apatite induction by insoluble dentin collagen," Journal of Bone and Mineral Research, vol. 13, no. 2, pp. 265-270, 1998.

[19] T. Saito, M. Yamauchi, Y. Abiko, K. Matsuda, and M. A. Crenshaw, "In vitro apatite induction by phosphophoryn immobilized on modified collagen fibrils," Journal of Bone and Mineral Research, vol. 15, no. 8, pp. 1615-1619, 2000.

[20] J. Jadlowiec, H. Koch, X. Zhang, P. G. Campbell, M. Seyedain, and C. Sfeir, "Phosphophoryn regulates the gene expression and differentiation of NIH3T3, MC3T3-E1, and human mesenchymal stem cells via the integrin/MAPK signaling pathway," Journal of Biological Chemistry, vol. 279, no. 51, pp. 53323-53330, 2004.

[21] J. A. Jadlowiec, X. Zhang, J. Li, P. G. Campbell, and C. Sfeir, "Extracellular matrix-mediated signaling by dentin phosphophoryn involves activation of the Smad pathway independent of bone morphogenetic protein," Journal of Biological Chemistry, vol. 281, no. 9, pp. 5341-5347, 2006.

[22] Y. Yasuda, M. Izumikawa, K. Okamoto, T. Tsukuba, and T. Saito, "Dentin phosphophoryn promotes cellular migration of human dental pulp cells," Journal of Endodontics, vol. 34, no. 5, pp. 575578, 2008.

[23] W. A. Butler, "Dentin-specific proteins," Methods in Enzymology, vol. 145, pp. 290-303, 1987.

[24] A. Lihme, C. Schafer-Nielsen, and K. P. Larsen, "Divinylsulphone-activated agarose. Formation of stable and nonleaking affinity matrices by immobilization of immunoglobulins and other proteins," Journal of Chromatography-Biomedical Applications, vol. 376, pp. 299-305, 1986.

[25] A. A. Baykov, O. A. Evtushenko, and S. M. Avaeva, "A malachite green procedure for orthophosphate determination and its use in alkaline phosphatase-based enzyme immunoassay," Analytical Biochemistry, vol. 171, no. 2, pp. 266-270, 1988.

[26] K. Hayashi, K. Handa, T. Koike, and T. Saito, "The possibility of genistein as a direct pulp capping agent," Dental Materials Journal, vol. 32, no. 6, pp. 976-985, 2013.

[27] I. Mejare, "Endodontics in primary teeth," in Textbook of Endodontology, G. Bergenholtz, P. Horsted-Bindslev, and C. Reit, Eds., pp. 102-119, 2003.

[28] M. Nakashima, "Induction of dentin formation on canine amputated pulp by recombinant human bone morphogenetic proteins (BMP)-2 and -4," Journal of Dental Research, vol. 73, no. 9, pp. 1515-1522, 1994.

[29] R. B. Rutherford, L. Spångberg, M. Tucker, D. Rueger, and M. Charette, "The time-course of the induction of reparative dentine formation in monkeys by recombinant human osteogenic protein-1," Archives of Oral Biology, vol. 39, no. 10, pp. 833-838, 1994.

[30] M. Goldberg, N. Six, F. Decup et al., "Application of bioactive molecules in pulp-capping situations," Advances in Dental Research, vol. 15, pp. 91-95, 2001.

[31] F. Decup, N. Six, B. Palmier et al., "Bone sialoprotein-induced reparative dentinogenesis in the pulp of rat's molar," Clinical oral investigations, vol. 4, no. 2, pp. 110-119, 2000.

[32] A. Gericke, C. Qin, Y. Sun et al., "Different forms of DMP1 play distinct roles in mineralization," Journal of Dental Research, vol. 89, no. 4, pp. 355-359, 2010. 
[33] N. Six, D. Septier, C. Chaussain-Miller, R. Blacher, P. DenBesten, and M. Goldberg, "Dentonin, a MEPE fragment initiates pulphealing response to injury," Journal of Dental Research, vol. 86, no. 8, pp. 780-785, 2007.

[34] Y. Nakamura, L. Hammarström, E. Lundberg et al., "Enamel matrix derivative promotes reparative processes in the dental pulp," Advances in Dental Research, vol. 15, pp. 105-107, 2001.

[35] T. Okiji and K. Yoshiba, "Reparative dentinogenesis induced by mineral trioxide aggregate: a review from the biological and physicochemical points of view," International Journal of Dentistry, vol. 2009, Article ID 464280, 12 pages, 2009.

[36] M. L. R. Accorinte, A. D. Loguercio, A. Reis et al., "Response of human dental pulp capped with MTA and calcium hydroxide powder," Operative Dentistry, vol. 33, no. 5, pp. 488-495, 2008.

[37] P. Bornstein and E. H. Sage, "Matricellular proteins: extracellular modulators of cell function," Current Opinion in Cell Biology, vol. 14, no. 5, pp. 608-616, 2002.

[38] M. Goldberg, J. Farges, S. Lacerda-Pinheiro et al., "Inflammatory and immunological aspects of dental pulp repair," Pharmacological Research, vol. 58, no. 2, pp. 137-147, 2008.

[39] S. A. Eming, T. Krieg, and J. M. Davidson, "Inflammation in wound repair: molecular and cellular mechanisms," Journal of Investigative Dermatology, vol. 127, no. 3, pp. 514-525, 2007.

[40] M. Goldberg and A. Smith, "Cells and extracellular matrices of dentin and pulp. A biological basis for repair and tissue engineering," Critical Reviews in Oral Biology \& Medicine, vol. 15, no. 1, pp. 13-27, 2004.

[41] M. Jontell, G. Bergenholtz, A. Scheynius, and W. Ambrose, "Dendritic cells and macrophages expressing class II antigens in the normal rat incisor pulp," Journal of Dental Research, vol. 67, no. 10, pp. 1263-1266, 1988.

[42] S. Nishikawa and F. Sasaki, "Apoptosis of dental pulp cells and their elimination by macrophages and MHC class II-expressing dendritic cells," Journal of Histochemistry and Cytochemistry, vol. 47, no. 3, pp. 303-311, 1999.

[43] M. Jontell, T. Okiji, U. Dahlgren, and G. Bergenholtz, "Immune defense mechanisms of the dental pulp," Critical Reviews in Oral Biology and Medicine, vol. 9, no. 2, pp. 179-200, 1998.

[44] K. Tanaka, T. Goto, T. Miyazaki, Y. Morita, S. Kobayashi, and T. Takahashi, "Apatite-coated hyaluronan for bone regeneration," Journal of Dental Research, vol. 90, no. 7, pp. 906-911, 2011.

[45] Y. A. Petrenko, R. V. Ivanov, A. Y. Petrenko, and V. I. Lozinsky, "Coupling of gelatin to inner surfaces of pore walls in spongy alginate-based scaffolds facilitates the adhesion, growth and differentiation of human bone marrow mesenchymal stromal cells," Journal of Materials Science: Materials in Medicine, vol. 22, no. 6, pp. 1529-1540, 2011.

[46] X. Chevalier, J. Jerosch, P. Goupille et al., "Single, intra-articular treatment with $6 \mathrm{ml}$ hylan G-F 20 in patients with symptomatic primary osteoarthritis of the knee: a randomised, multicentre, double-blind, placebo controlled trial," Annals of the Rheumatic Diseases, vol. 69, no. 1, pp. 113-119, 2010.

[47] G. Marcì, G. Mele, L. Palmisano, P. Pulito, and A. Sannino, "Environmentally sustainable production of cellulose-based superabsorbent hydrogels," Green Chemistry, vol. 8, no. 5, pp. 439-444, 2006. 

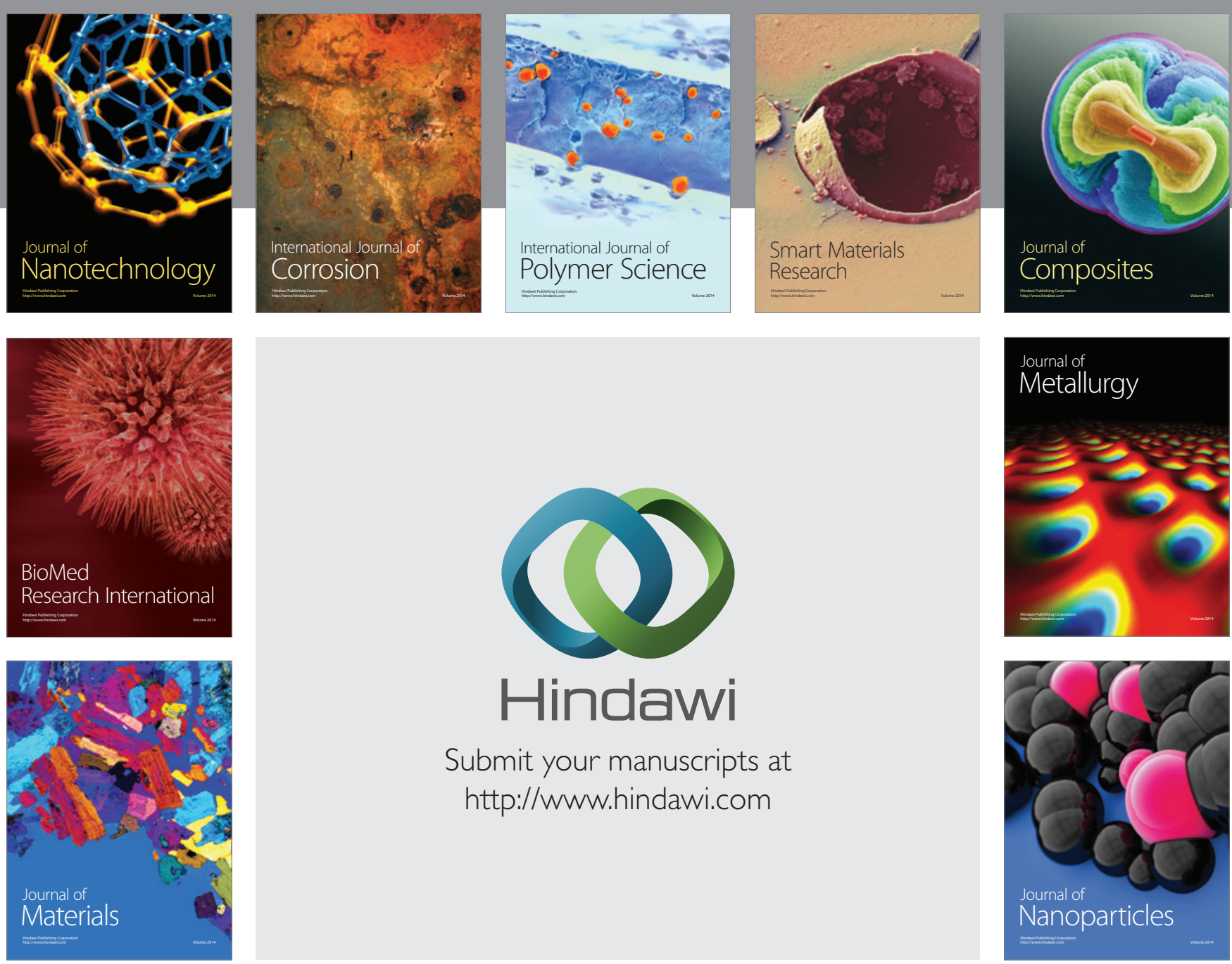

Submit your manuscripts at http://www.hindawi.com
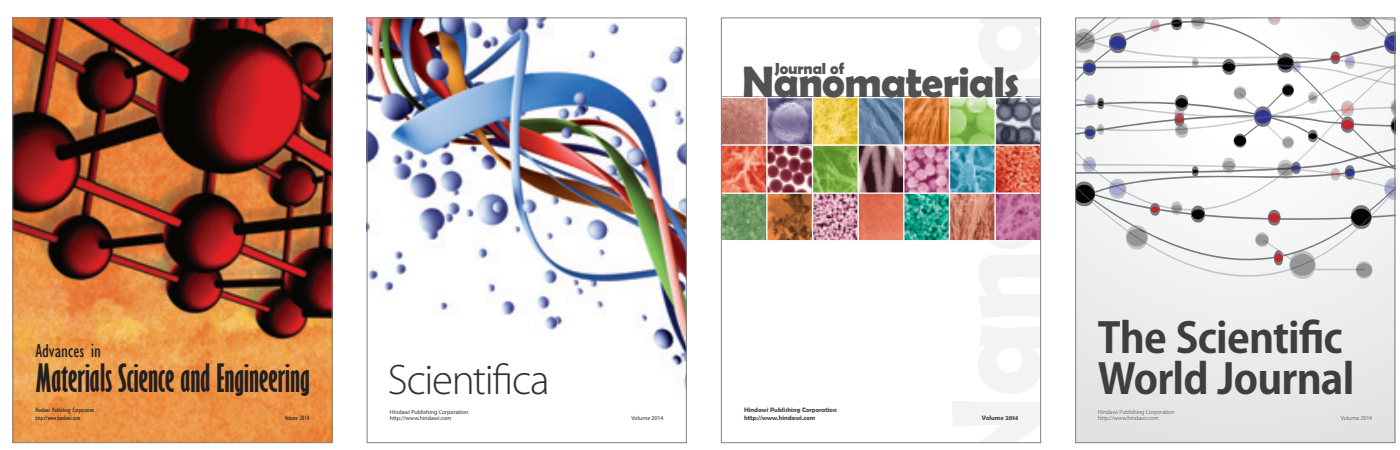

\section{The Scientific World Journal}
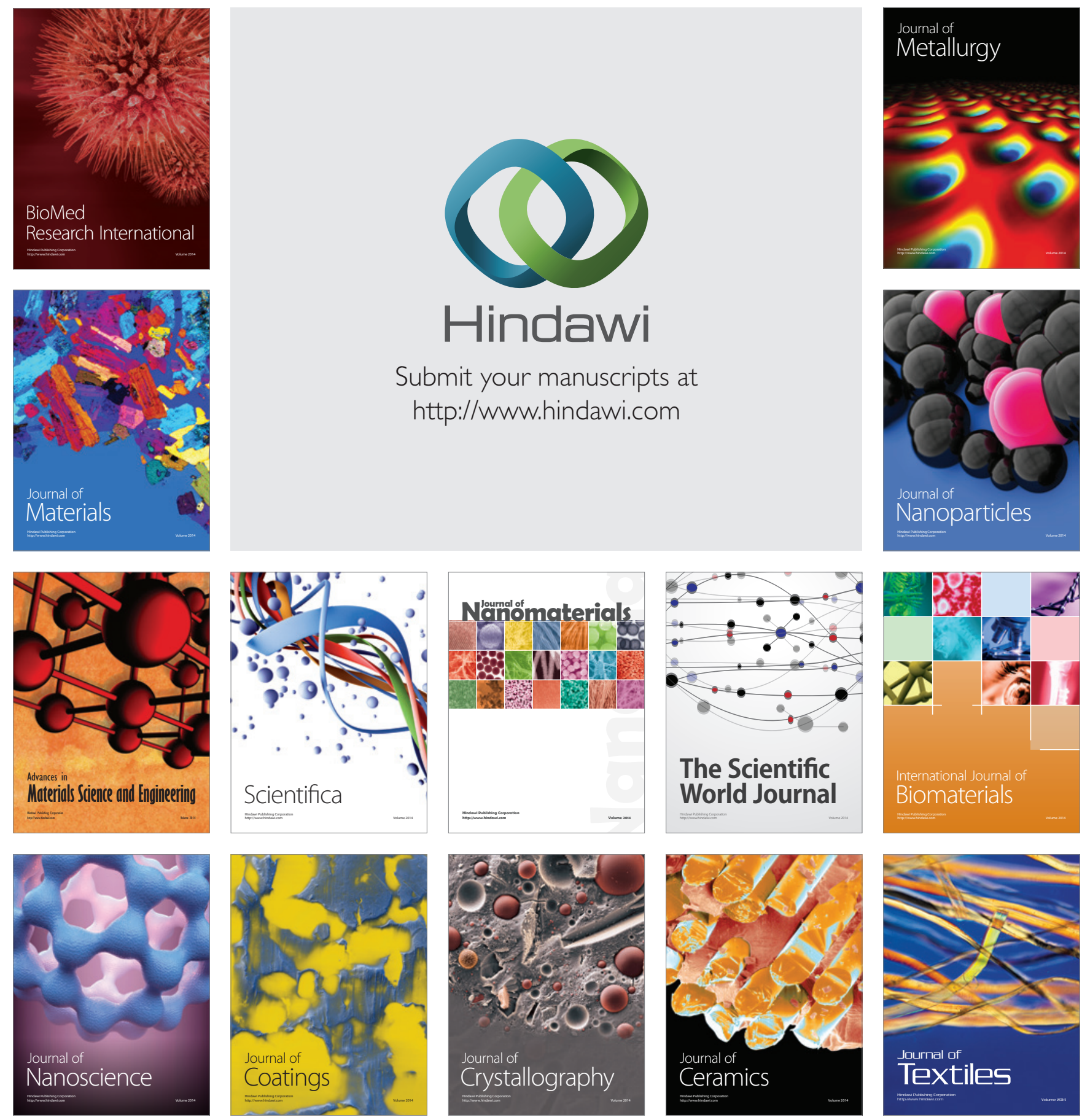